\title{
Magnetic Poles Determinations and Robustness of Memory Effect Upon Solubilization in a Dy"l'-based Single Ion Magnet
}

\author{
Tamyris T. da Cunha, ${ }^{1,3}$ Julie Jung, ${ }^{1}$ Marie-Emmanuelle Boulon, ${ }^{2}$ Giulio Campo, ${ }^{2}$ Fabrice Pointil- \\ lart, ${ }^{, 1}$ Cynthia L. M. Pereira, ${ }^{3}$ Boris Le Guennic, , ${ }^{* 1}$ Olivier Cador, ${ }^{*}, 1$ Kevin Bernot,4 Francesco Pin- \\ eider, ${ }^{2,5}$ Stéphane Golhen ${ }^{1}$ and Lahcène Ouahab ${ }^{1}$ \\ ${ }^{1}$ Institut des Sciences Chimiques de Rennes, UMR 6226 CNRS - Université de Rennes 1, 263 Avenue du Général Le- \\ clerc 35042 Rennes Cedex (France) \\ ${ }^{2}$ Department of Chemistry “Ugo Schiff” and INSTM RU, University of Florence, 50019 Sesto Fiorentino (Italy) \\ 3 Departamento de Química, Instituto de Ciências Exatas, Universidade Federal de Minas Gerais, 31270-901 Belo \\ Horizonte, MG (Brazil) \\ ${ }^{4}$ Université Européenne de Bretagne, INSA, ISCR, UMR 6226, 35708 Rennes (France) \\ ${ }_{5}$ CNR-ISTM c/o Department of Chemistry, University of Padova, 35131 Padova (Italy) \\ Supporting Information Placeholder
}

\begin{abstract}
The $\left[\mathrm{Dy}(\mathrm{tta})_{3}(\mathrm{~L})\right]$ complex behaves as a single ion magnet both in crystalline phase and in solution. Experimental and theoretical magnetic anisotropy axes perfectly match and lie along the most electro-negative atoms of the coordination sphere. Both VSM and MCD measurements highlight the robustness of the complex with the persistence of the memory effect even in solution up to $4 \mathrm{~K}$.
\end{abstract}

Molecular magnetism has retained the attention of the scientific community for more than two decades with, in particular, the discovery of single molecule magnets (SMMs). ${ }^{1}$ Key ingredients to build SMMs are a large magnetic moment and a strong magnetic anisotropy. To satisfy the first requisite, a strategy consists in coupling first row transition ions but at the expense of the magnetic anisotropy. ${ }^{2}$ If lanthanides or actinides are used instead, the advantage of their greater magnetic anisotropy is counter-balanced by their poor ability to couple magnetically. ${ }^{3-5}$ Moreover, the orientation of the easy magnetization axis with respect to the molecular architecture is also a crucial characteristic to determine the magnetic poles and understand the lines of force driving the SMM behavior. Finally, on a more fundamental point of view, the presence of a magnetic hysteresis that is thought to have a molecular origin remains the strictest criterion to certify that an isolated molecule can operate as a magnet.

Recently, a magnetic hysteresis has been observed for molecules wired to a gold surface ${ }^{6}$ or grafted on single-wall carbon nanotubes7. This clearly demonstrates the feasibility for an isolated molecule to store the information, which is of paramount importance to integrate such systems in useful devices. Nevertheless, in these examples the memory effect emerges only at the sub-Kelvin scale. Thus, the opening of a hysteresis loop at temperatures accessible with standard cryogenic techniques remains a challenge for isolated molecules. In the case of lanthanide-based SMMs, several exam- ples of magnetic hysteresis at $2 \mathrm{~K}$ are reported for molecules in a crystalline phase ${ }^{1,5,8,9}$ with a record at $14 \mathrm{~K}^{10}$ In some cases, hysteresis is observed on paramagnetic complexes diluted in a diamagnetic crystal latticegg,h that again limits the degrees of freedom so that the observed magnetic behaviors are attributed to a well-defined motionless molecular geometry. A contrario, with the exception of Magnetic Circular Dichroism (MCD) studies of $\left[\mathrm{Pc}_{2} \mathrm{~Tb}\right]$ in its oxidized, neutral and reduced states ${ }^{11}$ and SQUID investigations of trivalent uranium complexes ${ }^{12}$, only 3 d-based clusters have been considered for memory effects in solution. However, the uniqueness of the memory effect independently of the medium in which the molecule is immerged represents one of the fundamental characteristics of a SMM. In this line, some of us recently investigated the magnetic properties of mononuclear Dy ${ }^{\mathrm{III}}$ complexes, [Dy(hfac) $\left.)_{3}(\mathrm{~L})\right] \quad\left(\mathrm{hfac}=1,1,1,5,5,5^{-}\right.$ hexafluoroacetylacetonate; L=TTF-based ligands where TTF=tetrathiafulvalene) in both solid-state and frozen solution. ${ }^{13}$ Strikingly, whereas SMM behavior was observed in solution, it vanishes in the solid-state because of intermolecular interactions (hydrogen-bonds). On the other hand, the behaviors observed in the solid state are not often transferred in solution due to the higher number of degrees of freedom. To dispose of such inconvenience, we now propose to replace the $\mathrm{hfac}^{-}$anions by $\mathrm{tta}^{-} \quad\left(\mathrm{tta}^{-}=\mathbf{2}^{-}\right.$ thenoyltrifluoroacetonate) to stabilize the molecular structure and to improve the performance of the SMM in reorganizing the charge density in the first coordination sphere of Dy ${ }^{\text {III }}$, as suggested recently.14,15 Indeed, thiophene moieties are less electro-attractive than $-\mathrm{CF}_{3}$ groups and so, the negative charges on the oxygen atoms of the diketonate ligands are more important in the tta- derivative.

In this article, we present the synthesis and crystal structure of a mononuclear Dy ${ }^{\mathrm{III}}$-based complex, namely $\left[\mathrm{Dy}(\mathrm{tta})_{3}(\mathrm{~L})\right]$ (Figure 1) where $\mathbf{L}=4,5$-bis(propylthio)-tetrathiafulvalene-2(2-pyridyl)benzimidazole-methyl-2-pyridine. The magnetic properties of this complex were investigated both in solid 
state and frozen solution. We demonstrate that the complex behaves as a SMM in both media with characteristic magnetic hysteresis loops. Single-crystal magnetic measurements coupled with $a b$ initio calculations allow the determination of the principal magnetic axes and the interpretation of the characteristics of this SMM.

$\left[\mathrm{Dy}(\mathrm{tta})_{3}(\mathbf{L})\right]$ complex was synthesized by reacting ligand $\mathbf{L}$ and tris(2-thenoyltrifluoroacetonate)bis(aqueous)Dy ${ }^{\mathrm{III}}$ in $\mathrm{CH}_{2} \mathrm{Cl}_{2}$. $n$-hexane slow diffusion in the mother solution affords red single crystals that are stable under aerobic conditions and suitable for X-ray diffraction analysis (Figures $\mathrm{S}_{1}, \mathrm{~S}_{2}$ and Tables $\mathrm{S}_{1}, \mathrm{~S}_{2}$ ). Dy ${ }^{\mathrm{III}}$ ion is in a $\mathrm{N}_{2} \mathrm{O}_{6}$ square antiprism environment $\left(D_{4 d}\right.$ symmetry) made of six oxygen and two nitrogen atoms that belong to three tta- anions and one $\mathbf{L}$ ligand, respectively (Figure 1 ). Central $\mathrm{C}=\mathrm{C}$ bond length of the TTF core $(1.343(9) \AA$ ) ) attests for the neutrality of the (2pyridyl)benzimidazole moiety. Crystal packing is governed by "head-to-tail" arrangement of $\mathbf{L}$. The resulting dimers create $\pi-\pi$ interactions through the tta- anions (see Figure S2). The shortest intermolecular Dy-Dy distance is $9.447 \AA$ and each Dy ${ }^{\mathrm{III}}$ ion can be considered as isolated.

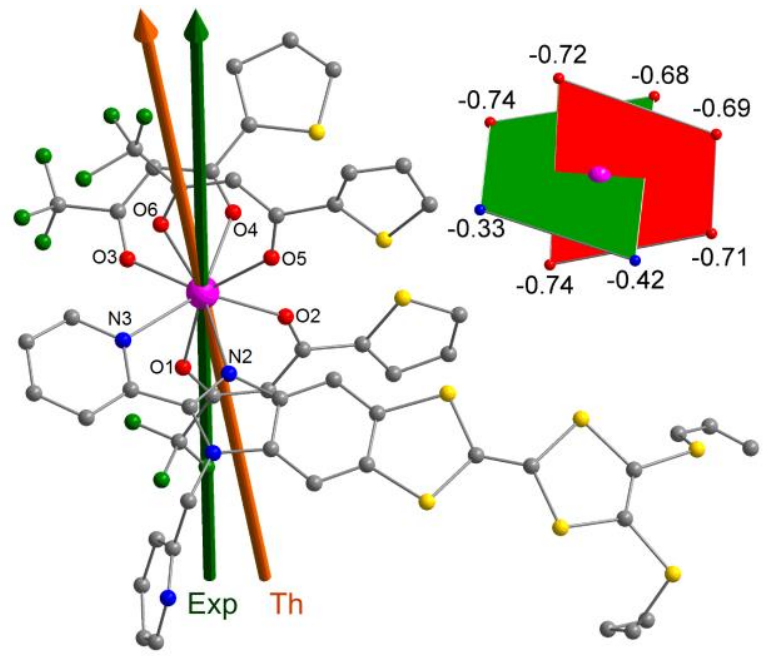

Figure 1. Representation of the crystallographic structure of $\left[\right.$ Dy $\left.(\mathrm{tta})_{3}(\mathbf{L})\right] \cdot \mathrm{C}_{6} \mathrm{H}_{14}(\mathrm{H}$ atoms and solvent molecule omitted for clarity). Pink, Dy; green, F; yellow, S; gray, C; blue, N; red, O. Experimental (dark green) and theoretical (orange) anisotropy axis. Inset: scheme of Dy ${ }^{\mathrm{III}}$ first coordination sphere with calculated charges and the two perpendicular planes formed by the negative charges (see text).

Dc magnetometry (Figure $S_{3}$ ) was performed from 2 to 300 $\mathrm{K}$. Room temperature value of $\chi_{M} T\left(14 \mathrm{~cm}^{3} \mathrm{~K} \mathrm{~mol}^{-1}, \chi_{M}\right.$ being the molar magnetic susceptibility and $T$ the temperature in Kelvin) agrees with the expected one for an isolated DyII. The $\chi_{M} T$ vs $T$ curve decreases monotonically to reach $11.5 \mathrm{~cm}^{3}$ $\mathrm{K} \mathrm{mol}^{-1}$ at $2 \mathrm{~K}$. At $2 \mathrm{~K}$, the $M$ vs $H$ magnetization saturates at 5 $\mathrm{N} \beta$ which agrees with a pure Ising $M_{J}= \pm 15 / 2$ ground state. To investigate the Ising character of the molecular magnetic moment, angular resolved magnetometry was undertaken on a single crystal of $\left[\mathrm{Dy}(\mathrm{tta})_{3}(\mathbf{L})\right] \cdot \mathrm{C}_{6} \mathrm{H}_{14}$. Angular dependence of the magnetization was measured in three orthogonal planes of an oriented single-crystal. Rotations were found to be temperature independent (Figure $\mathrm{S}_{4}$ ) and molar magnetic susceptibility was fitted with:

$\chi_{M}=M / H=\chi_{\alpha \alpha} \cos ^{2} \theta+\chi_{\beta \beta} \sin ^{2} \theta+2 \chi_{a \beta} \sin \theta \cos \theta$ where $\alpha$ and $\beta$ are the directions $\mathrm{X}, \mathrm{Y}$ and $\mathrm{Z}$ (Figures 2 and $\mathrm{S}_{5}$ ) in a cyclic permutation and $\theta$ is the angle between $H$ and $\alpha$. In the effective spin $1 / 2$ formalism, the largest principal value of the Zeeman tensor is equal to 18.65 close to the expected value (20.00) for a purely axial magnetic moment. More interesting is the orientation of the experimental easy axis with respect to the molecular topology. This axis is almost collinear to the $C_{4}$ axis, i.e. the normal of the plane formed by the Dy ${ }^{\mathrm{III}}$ ion and the two nitrogen atoms of the imidazole-pyridine rings and one tta- ligand (green plane on Figure 1). Oxygen atoms are formally more negatively charged than nitrogen atoms owing to the intrinsic charge of the tta- ligand and the electro-negativity of oxygen. Then, in solely considering the first coordination sphere of Dy ${ }^{\mathrm{III}}$, the $\left(\mathrm{O}_{1}, \mathrm{O}_{2}, \mathrm{O}_{5}, \mathrm{O} 6\right)$ pseudo-plane should be more negatively charged than the quasi-perpendicular $\left(\mathrm{N}_{2}, \mathrm{~N}_{3}, \mathrm{O}_{3}, \mathrm{O}_{4}\right)$ pseudo-plane. If one considers Dy ${ }^{\mathrm{III}}$ as an oblate ion, and following qualitative arguments based on the aspherical electron density distributions of lanthanide ions, ${ }^{14,15}$ the largest $M_{J}$ should be stabilized along the more negatively charged direction that is the fully oxygenated $\left(\mathrm{O}_{1}, \mathrm{O}_{2}, \mathrm{O}_{5}, \mathrm{O} 6\right)$ plane. This is in agreement with what is experimentally observed here.

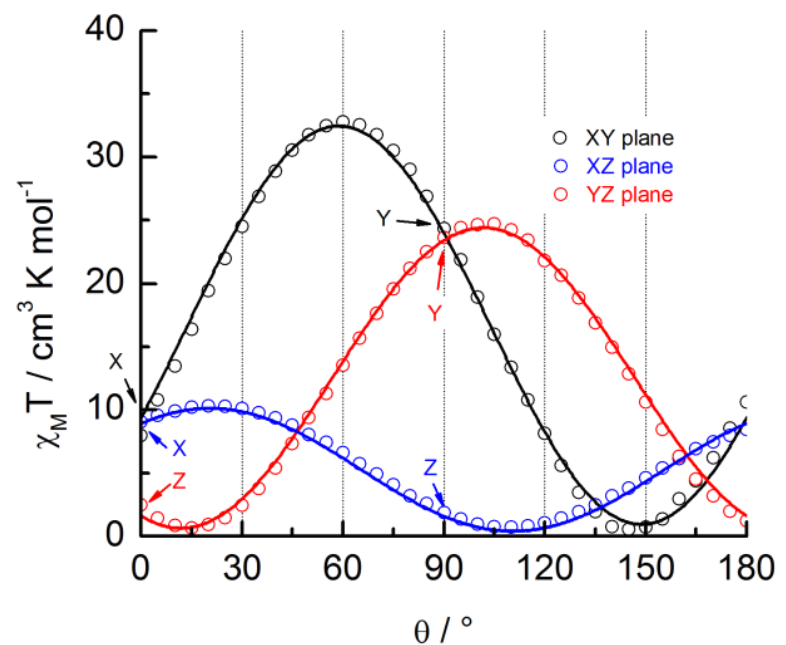

Figure 2. Angular dependence of $\chi_{M} T$ of a single crystal rotating in three perpendicular planes with $H=1 \mathrm{kOe}$ at $2 \mathrm{~K}$ (see SI for plane definitions). Best fitted curves in full lines.

To go beyond this qualitative interpretation, CASSCF/RASSISO calculations were carried out on the complete molecular structure of $\left[\mathrm{Dy}(\mathrm{tta})_{3}(\mathrm{~L})\right]$ (see computational details in SI). ${ }^{16}$ Energy spectrum and $g$-tensors for the eight Kramers doublets of the ground ${ }^{6} \mathrm{H}_{15 / 2}$ multiplet of the Dy ${ }^{\mathrm{III}}$ ion are given in Table $\mathrm{S}_{3}$. Calculations confirm the strong axiality of the ground Kramers doublet with a large $g_{z}$ value (19.50) close to the expected $g_{z}=20$ for a pure $M_{J}= \pm 15 / 2$. The $g$-tensor orientation of the first excited state, located at $+126 \mathrm{~cm}^{-1}$, does not deviate significantly from that of the ground state even if the $g_{z}$ value is much smaller (15.3). Furthermore, both $\chi_{M} T v s$. $T$ and $M$ vs. $H$ curves are fairly well reproduced (Figure $S_{3}$ ). The calculated ground state easy axis (Figure 1) is almost parallel to the experimental one, with a small deviation of 7.6. This excellent agreement between experimental and computed anisotropy axis, a comparison that is still scarce in the literature, ${ }^{17,18}$ gives us confidence in the subsequent quantitative magneto-structural analysis. To this purpose, atomic charges were calculated at the CASSCF level and reproduced 
in Figure 1. As expected, the negative charges on the oxygen atoms are substantially higher than on the nitrogen ones, inducing the electrostatic anisotropy that governs the orientation of the easy axis.

The out-of-phase component of the ac susceptibility ( $\chi$ ") of $\left[\mathrm{Dy}(\mathrm{tta})_{3}(\mathbf{L})\right] \cdot \mathrm{C}_{6} \mathrm{H}_{14}$ immobilized powder shows a frequency dependence in the $1.8-15 \mathrm{~K}$ temperature range (external dc field $\mathrm{H}_{\mathrm{dc}}=\mathrm{o}$ ) (top of Figure 3 and Figure S6). Relaxation time $\tau$ extracted using an extended Debye model (Table $\mathrm{S}_{4}$ ) follows a combination of thermally activated and temperature independent regimes: $\tau^{-1}=\tau_{\mathrm{o}}^{-1} \exp (-\Delta / T)+\tau_{\mathrm{TI}}{ }^{-}$(where $\Delta$ is the energy barrier) between 1.8 and $15 \mathrm{~K}$ (see Figure 4 and Table S6) and $\tau_{\mathrm{TI}}=1.62( \pm 0.04) \times 10^{-3} \mathrm{~s}$ (Figure $\left.\mathrm{S}_{7}\right)$. In order to minimize the number of fitted parameters, the low frequency limit was fixed to its dc value. The temperature independent regime supports the idea that a direct relaxation process between degenerated Kramers doublets of the ${ }^{6} \mathrm{H}_{15 / 2}$ multiplet operates. The application of a moderate field at $2 \mathrm{~K}$ does not shift the maximum of $\chi$ " to lower frequency but instead splits the relaxation into two well separated processes: a slow relaxation (SR) and a fast relaxation (FR, Figure S8). For $H_{d c}>500 \mathrm{Oe}$, the FR process totally disappears and the whole relaxation occurs through the SR one. This feature was reported already by us and others for diverse mononuclear dysprosium-based SMMs. ${ }^{13,19}$ This feature is even visible when $\chi$ " is measured for a single crystal of $\left[\mathrm{Dy}(\mathrm{tta})_{3}(\mathrm{~L})\right] \cdot \mathrm{C}_{6} \mathrm{H}_{14}$ oriented with the magnetic field parallel to the easy magnetic axis (Figure S9).

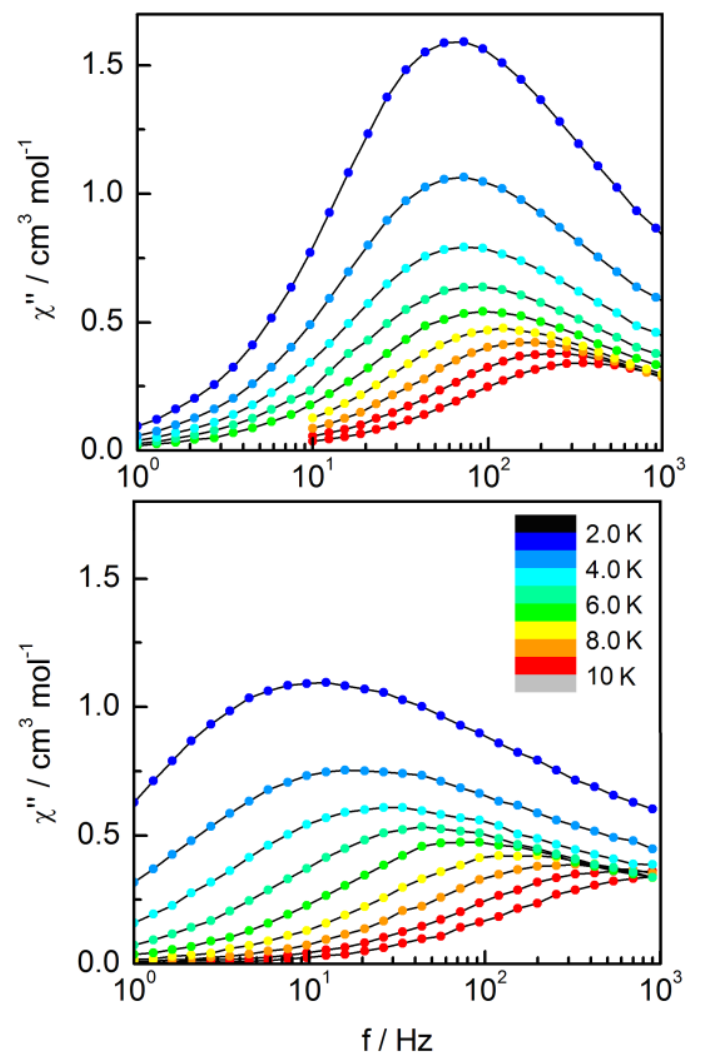

Figure 3. Frequency dependences of the out-of-phase components of the ac susceptibility in zero field for the solid state sample (top) and a frozen solution (bottom) of $\left[\mathrm{Dy}(\mathrm{tta})_{3}(\mathbf{L})\right]$ measured at various temperatures.
Measurement for $H_{d c}=1 \mathrm{kOe}$, shows that relaxation time follows an Arrhenius law between 14 and $7 \mathrm{~K}$ (Table $\mathrm{S}_{5}$ ) with an activation energy (Table S6) similar to $H_{d c}=0$. A fundamental question must be addressed at this stage: "is the observed SMM behavior intrinsic to the molecule or due to the crystalline solid-state?" In order to study the pure molecular character of the dynamic magnetic properties of $\left[\mathrm{Dy}(\mathrm{tta})_{3}(\mathbf{L})\right]$, a dichloromethane solution was prepared. UVVis absorption measurements and TD-DFT calculations (performed on the $\mathrm{Y}^{\mathrm{III}}$ diamagnetic analogue) highlight a red shift $\left(2100 \mathrm{~cm}^{-1}\right)$ of the lowest absorption band identified as an $\mathrm{HOMO} \rightarrow$ LUMO intra-ligand charge transfer (ILCT) in $\left[\mathrm{Dy}(\mathrm{tta})_{3}(\mathrm{~L})\right]\left(23200 \mathrm{~cm}^{-1}\right)$ compared to $\mathbf{L}\left(25300 \mathrm{~cm}^{-1}\right)$ attesting for the stability of the complex in a $(7.7 \mathrm{mM})$ dichloromethane solution (Figures S1o and S11 and Table S7). Dynamic magnetic behavior of the solution is close to what is observed in solid-state (Figures 3 and S12). However two small differences are visible: i) $\chi$ ” vs. $f$ curves are broader in solution and ii) thermally independent regime of the frozen solution is slightly slower (Table S6 and Figure 4). The former may be the consequence of a large distribution of slightly different coordination polyhedron symmetries in solution which leads to magnetic species relaxing at different frequencies as attested with the dramatically larger parameter $\alpha$ in solution when compared with solid-state. The latter can be explained by the fact that whereas in the condensed phase the molecules are close enough $(\sim 10 \AA)$ to generate an internal dipolar field, the average distance between the molecules is calculated to be around $60 \AA$ in the frozen solution. Thus the dipolar field that is expected to accelerate the relaxation is no longer efficient.

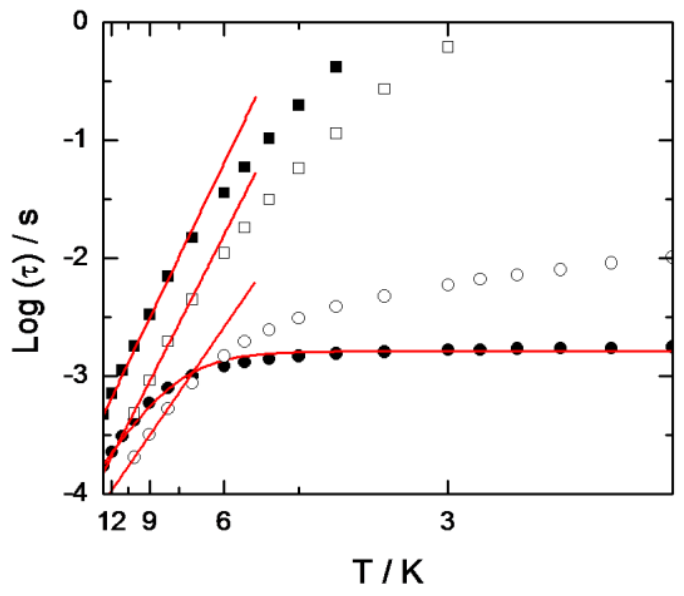

Figure 4. Log scale plots of the temperature dependence of the relaxation time of $\left[\mathrm{Dy}(\mathrm{tta})_{3}(\mathbf{L})\right] \cdot \mathrm{C}_{6} \mathrm{H}_{14}$ in solid-state (full symbols) and a frozen dichloromethane solution of $\left[\mathrm{Dy}(\mathrm{tta})_{3}(\mathbf{L})\right]$ (empty symbols) measured with $H_{d c}=0$ (circles) and $H_{d c}=1 \mathrm{kOe}$ (squares). Red lines correspond to the best fitted curves with Arrhenius or modified Arrhenius laws (see text).

The substitution of the hfac- ancillary anions by tta- ones enhances the dynamic characteristic of the SMM as the energy barrier is doubled (about $18 \mathrm{~K}^{13}$ vs $40 \mathrm{~K}$ ). This behavior can be correlated to the higher symmetry of the coordination polyhedron in $\left[\mathrm{Dy}(\mathrm{tta})_{3}(\mathrm{~L})\right] \cdot \mathrm{C}_{6} \mathrm{H}_{14}\left(\mathrm{D}_{4 \mathrm{~d}}\right)$ than the one in $\left[\mathrm{Dy}(\mathrm{hfac})_{3}(\mathbf{L})\right]\left(\mathrm{C}_{2 \mathrm{v}}\right)$. 


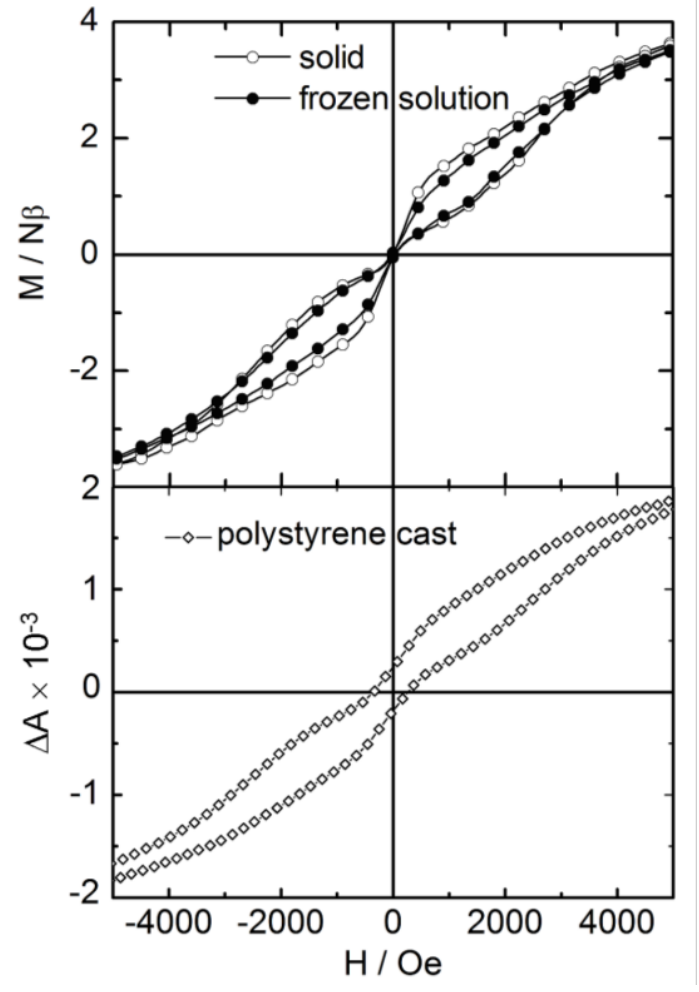

Figure 5. (Bottom) Hysteresis loops measured at $2 \mathrm{~K}$ at 150 $\mathrm{Oe} \mathrm{s}^{-1}$ on a solid sample and in frozen solution. (Below) MCD hysteresis loop measured at $27400 \mathrm{~cm}^{-1}(365 \mathrm{~nm})$ at $1.5 \mathrm{~K}$ and $150 \mathrm{Oe} \mathrm{s}^{-1}$ on a polystyrene solid solution.

Moreover most of the solution's molecules are involved in the slow relaxation as the non-relaxing fraction of the magnetization tends to zero (Table S8). These crucial results attest that magnetic slow relaxation of $\left[\mathrm{Dy}(\mathrm{tta})_{3}(\mathbf{L})\right]$ is of molecular origin. This behavior is highlighted in VSM (Vibrating Sample Magnetometry) (Figures 5, $\mathrm{S}_{13}$ and $\mathrm{S}_{14}$ ) and MPMS (Figure S15) hysteresis measurements both on solidstate sample and frozen solution. Fast tunneling in zero-field strangle the loops that take the classical butterfly shape. At a sweeping rate of $150 \mathrm{Oe} \mathrm{s}^{-1}$ and at $2 \mathrm{~K}$ the maximum coercive field is close to 700 Oe in solid-state and 500 Oe in solution. For both samples the magnetic irreversibility is centered at 1 kOe (Figure 5). The two curves almost superimpose. The hysteresis loop measured on the frozen solution is concentration independent (Figure S14) meaning that the width of the hysteresis is not related to the distance between the magnetic centers, which again underlines the molecular origin of the magnetization dynamics. Finally, to further investigate the molecular nature of the observed hysteresis we performed MCD measurements on a solid solution (18.4 $\mathrm{mM})$ of $\left[\mathrm{Dy}(\mathrm{tta})_{3}(\mathbf{L})\right]$ in polystyrene cast as a thin film on a glass slide. MCD is able to selectively probe the dissolved material in the sample, since the technique is intrinsically insensitive to aggregates, making it a very useful tool to study diluted SMMs. ${ }^{20-22}$ Hysteresis loops recorded at various temperatures at $27400 \mathrm{~cm}^{-1}$ (HOMO-2/-5 $\rightarrow$ LUMO ILCT, Table $\mathrm{S}_{7}$, Figures $\mathrm{S}_{10}$ and $\mathrm{S}_{11}$ ) show the expected temperature dependence of the opening of the hysteresis (Figure S16) with the shape of the hysteresis loops similar to what is observed in solution.

Here we demonstrated that, $\left[\mathrm{Dy}(\mathrm{tta})_{3}(\mathrm{~L})\right]$ is a remarkable single ion magnet whose hysteretic behavior is measureable for the first time both in solid-state and solution. Experimental and theoretical investigations confirm the axial hard direction of the DyIII ion close to the pseudo $C_{4}$ symmetry axis of the coordination polyhedron while the equatorial plane corresponds to an easy plane of magnetization. By rational molecular design, we managed to enhance the magnetic properties of the $\left[\mathrm{Dy}(\mathrm{tta})_{3}(\mathbf{L})\right]$ complex; indeed, the substitution of $\mathrm{hfac}^{-}$ancillary ligands with tta-leads to a greater energy barrier ( $\Delta$ has doubled) and in this compound the magnetic relaxation is so slow that the hysteresis is observed until $4 \mathrm{~K}$. This hysteretic behavior is evidenced thanks to VSM and MCD on both solid and solution highlighting the molecular origin of magnetization dynamics. The robustness of this magnetic behavior in various media turns out to be crucial to envision single-molecule magnet-based magnetic devices

\section{ASSOCIATED CONTENT}

\section{Supporting Information}

X-ray crystallographic files in CIF format, experimental and computational details, crystallographic data, and additional structural and magnetic figures and tables. This material is available free of charge via the Internet at http://pubs.acs.org.

\section{AUTHOR INFORMATION}

\section{Corresponding Author}

Fabrice.pointillart@univ-rennesı.fr

\section{Notes}

The authors declare no competing financial interests.

\section{ACKNOWLEDGMENT}

This work was supported by Région Bretagne, Rennes Métropole, INSA Rennes, CNRS, Université de Rennes 1 and FEDER. The financial support of the Italian MIUR through FIRB project Rete ItalNanoNet (RBPRo5 $\mathrm{JH}_{2} \mathrm{P}$ ) and of the European Commission through the ERC-AdG 267746 MolNanoMas (project n. 267746) and of Fondazione Cariplo through Project No. 2010-0612 is gratefully acknowledged.

\section{REFERENCES}

(1) Sessoli, R.; Gatteschi, D.; Caneschi, A.; Novak, M. A. Nature 1993, 365, 141-143.

(2) Gatteschi, D.; Sessoli, R.; Villain, J. Molecular nanomagnets, Oxford University Press, Oxford, 2006.

(3) Sessoli, R.; Powell, A. K. Coord. Chem. Rev. 2009, 253, 23282341.

(4] (a) Rinehart, J. D.; Long, J. R. J. Am. Chem. Soc. 2009, 131, 12558-12559. (b) Mougel, V.; Chatelain, L.; Pécaut, J.; Caciuffo, R.; Colineau, E.; Griveau, J.-C.; Mazzanti, M. Nature Chem. 2012, 4, 10111017

(5) Magnani, N.; Apostolidis, C.; Morgenstern, A.; Colineau, E.; Griveau, J.-C.; Bolvin, H.; Walter, O.; Caciuffo, R.; Angew. Chem. Int. Ed. 2011, 50, 1696-1698.

(6) (a) Mannini, M.; Pineider, F.; Danieli, C.; Totti, F.; Sorace, L.; Sainctavit, P.; Arrio, M.-A.; Otero, E.; Joly, L.; Cezar, J. C.; Cornia, A.; Sessoli, R. Nature 2010, 468, 417-421. (b) Mannini, M.; Pineider, F.; Sainctavit, P.; Danieli, C.; Otero, E.; Sciancalepore, C.; Talarico, A. M.; Arrio, M.-A.; Cornia, A.; Gatteschi, D.; Sessoli, R. Nature Materials, 2009, 8, 194-197.

(7) Giusti, A.; Charron, G.; Mazerat, S.; Compain, J. D.; Mialane, P.; Dolbecq, A.; Rivière, E.; Wernsdorfer, W.; Biboum, R. N.; Keita, 
B.; Nadjo, L.; Filoramo, A.; Bourgoin, J.-P.; Mallah, T. Angew. Chem. Int. Ed. 2009, 48, 4949-4952.

(8) Ishikawa, N.; Mizuno, Y.; Takamatsu, S.; Ishikawa, T.; Koshihara, S. Inorg. Chem. 2008, 47, 10217-10219.

(9) (a) Tian, H.; Wang, M.; Zhao, L.; Guo, Y.-N.; Guo, Y.; Tang, J.; Liu, Z. Chem. Eur. J. 2012, 18, 442-445. (b) Tian, H.; Zhao, L.; Guo, Y.N.; Guo, Y.; Tang, J.; Liu, Z. Chem. Commun. 2012, 48, 708-710. (c) Jiang, S.-D.; Wang, B.-W.; Sun, H.-L.; Wang, Z.-M.; Gao, S. J. Am. Chem. Soc. 2011, 133, 4730-4733. (d) Demir, S.; Zadrozny, J.-M.; Nippe, M.; Long, J. R. J. Am. Chem. Soc. 2012, 134, 18546-18549. (e) Cardona-Serra, S.; Clemente-Juan, J. M.; Coronado, E.; Gaita-Ariño, A.; Camón, A.; Evangelisti, M.; Luis, F.; Martínez-Pérez, M. J.; Sese, J. J. Am. Chem. Soc. 2012, 134, 14982-1499o. (f) Ganivet, C. R.; Ballesteros, B.; de la Torre, G.; Clemente-Juan, J. M.; Coronado, E.; Torres, T. Chem. Eur. J. 2013, 19, 1457-1465. (g) Bi, Y.; Guo, Y.-N.; Zhao, L.; Guo, Y.; Lin, S.-Y.; Jiang, S.-D.; Tang, J.; Wang, B.-W.; Gao, S. Chem. Eur. J. 2011, 17, 12476-12481. (h) Habib, F.; Lin, P.-H.; Long, J.; Korobkov, I.; Wernsdorfer, W.; Murugesu, M. J. Am. Chem. Soc. 2011, 133, 88308833.

(10) Rinehart, J. D.; Fang, M.; Evans, W. J.; Long, J. R. J. Am. Chem. Soc. 2011, 133, 14236-14239.

(11) Gonidec, M.; Davies, E. S.; McMaster, J.; Amabilino, D. B.; Veciana, J. J. Am. Chem. Soc. 2010, 132, 1756-1757.

(12) Moro, F.; Mills, D. P.; Liddle, S. T.; van Slageren, J. Angew. Chem. Int. Ed. 2013, 52, 3430-3433.

(13) Cosquer, G.; Pointillart, F.; Golhen, S.; Cador, O.; Ouahab, L. Chem. Eur. J. 2013, 19, 7895-7903.

(14) Rinehart, J. D.; Long, J. R. Chem. Sci. 2011, 2, 2078-2085.

(15) Chilton, N. F.; Langley, S. K.; Moubaraki, B.; Soncini, A.; Batten, S. R.; Murray, K. S. Chem. Sci. 2o13, 4, 1719-1730.

(16) Chibotaru, L ; F.; Ungur, L. J. Chem. Phys. 2012, 137, 064112.

(17) Cucinotta, G.; Perfetti, M.; Luzon, J.; Etienne, M.; Car, P.-E.; Caneschi, A.; Calvez, G.; Bernot, K.; Sessoli, R. Angew. Chem. Int. Ed. 2012, 51, 1606-1610.

(18) Boulon, M.-E.; Cucinotta, G.; Luzon, J.; Degl'Innocenti, C.; Perfetti, M.; Bernot, K.; Calvez, G.; Caneschi, A.; Sessoli, R. Angew. Chem. Int. Ed. 2013, 52, 350-354.

(19) Car, P.-E.; Perfetti, M.; Mannini, M.; Favre, A.; Caneschi, A.; Sessoli, R. Chem. Commun. 2011, 47, 3751-3753.

(20) McInnes, E. J. L.; Pidcock, E.; Oganesyan, V. S.; Cheesman, M. R.; Powell, A. K.; Thomson, A. J. J. Am. Chem. Soc. 2002, 124, 92199228.

(21) Bogani, L.; Cavigli, L.; Gurioli, M.; Novak, R. L.; Mannini, M.; Caneschi, A.; Pineider, F.; Sessoli, R.; Clemente-Leon, M.; Coronado, E.; Cornia, A.; Gatteschi, D. Adv. Mater. 2007, 19, 3906.

(22) Novak, R. L.; Pineider, F.; De Julian Fernandez, C.; Gorini, L.; Bogani, L.; Danieli, C.; Cavigli, L.; Cornia, A.; Sessoli, R. Inorg. Chim. Acta 2008, 361, 3970.

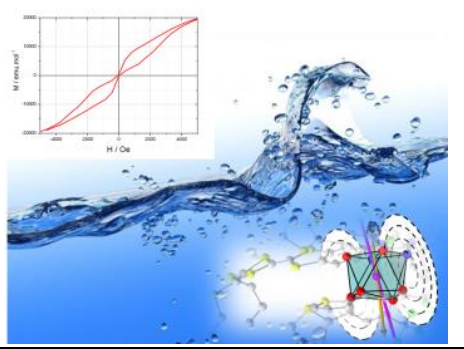

